\title{
Co-Morbidity and Medication Profiles of Patients with Epilepsy and Matched Controls in US and UK Electronic Health Records Systems
}

\author{
Lianna Ishihara and Michael Irizarry \\ GlaxoSmithKline PLC, \\ United Kingdom, \\ United States of America
}

\section{Introduction}

The global burden of epilepsy is substantial, especially in the developing regions of the world where treatments are less accessible (de Boer et al., 2008). One contribution to the overall disease burden is the higher prevalence of comorbidities among patients with epilepsy, compared to the general population. These include psychological comorbidities, such as depression, which have been associated with epilepsy, both before and after epilepsy diagnosis (Hesdorffer et al., 2005). Other neurological diseases such as migraine have also been associated with epilepsy (Ottman \& Lipton, 1994).

Two database studies were utilised to assess the overall comorbidity profiles of patients with epilepsy and matched controls without epilepsy. The analyses allow for comparisons between the relative prevalence estimates of comorbidities within populations captured through electronic health data sources. The US Impact National Managed Care Benchmark Database (IHCIS) is an insurance claims database provided by Ingenix, Eden Prairie, MN, and the UK General Practice Research Database (GPRD) Gold contains electronic medical records and is managed by the Medicines and Healthcare products Regulatory Agency.

The IHCIS database contains medical (inpatient, outpatient, physician, and ancillary) and pharmacy claims from a national sample of 46 managed care health plans covering approximately 93 million lives over the period 1997 to 2009.

The GPRD Gold is the world's largest computerised database of anonymised longitudinal medical records from primary care (Jick et al., 2003). The data are drawn from the computer systems used by general practitioners (GPs) to maintain the clinical records within their practices, and contain all records that primary care deemed relevant to patient care. Currently, data are available from 561 "research standard" general practices throughout the UK, providing information from 4.0 million currently registered patients (c. 9.0 million in total).

The objectives of the current analyses were: (1) to calculate the prevalence of epilepsy diagnosis in a US and a UK electronic health record system, stratified by gender and age 
category; (2) to describe and compare the prevalence of co-morbidities, in epilepsy patients and matched controls without any record of epilepsy; and (3) to describe the prevalence of medication use by epilepsy patients during a one-year period of observation.

\section{Methods}

This study was a case-control study comparing comorbidities between patients with epilepsy and matched controls without epilepsy, and a descriptive study reporting medication use among patients with epilepsy.

\subsection{Patient population}

\subsubsection{Case selection}

The definitions of active epilepsy reflected the structure of the databases and how medical and pharmacy data are captured:

In IHCIS, epilepsy cases were identified based on at least two records of epilepsy on or before the index date and with 30 days or more between the first and last record of epilepsy. At least one of the records of epilepsy had to be recorded within the lead-in period to identify individuals with recently active epilepsy. Epilepsy was defined as the ICD-9 code $345^{*}$, with * indicating all terms under this hierarchy. AED treatment was not part of the case definition of epilepsy in the IHCIS in order to capture epilepsy patients in the claims database that may not have pharmacy benefits. Also, disease diagnoses are recorded on a regular basis in the insurance claims, therefore a repeat diagnosis could be used to confirm the epilepsy diagnosis.

In the GPRD, epilepsy cases were identified as those with one epilepsy diagnosis (READ) code on or before the index date. The relevant GPRD medical codes were identified and reviewed by a clinician. Within the GPRD, active epilepsy cases were required to have at least 2 anti-epileptic drug (AED) prescriptions within -3 and +6 months of any epilepsy diagnosis code AND at least 1 AED prescription within the lead-in or analysis period. In the GPRD, a diagnosis is often recorded only one time on the database therefore the medications were used as a proxy to confirm the diagnosis and that the diagnosis was still active near the time of the index date.

Full definitions of index date, analysis and lead-in periods are provided in Section Data Analysis.

\subsubsection{Control selection}

Controls were matched to cases at a ratio of 1:1 by: gender, exact year of birth, duration of prior continuous enrolment in the database (from index date) by the following categories (1$<2$ years, $2-<3$ years, 3-<4 years, 4 or more years).

For IHCIS specifically, controls were also matched on pharmacy benefits throughout the full analysis period (Yes/No), Mental Health benefits on the index date (Yes/No/Partial), and Provider/Plan Type (on the index date).

For GPRD specifically, the controls were also matched by General Practice site.

\subsection{Data analysis}

The analysis period was defined as the 12 month period from 01/Jan/2009 to 31/Dec/2009, inclusive. The first day of the analysis period (01/Jan/2009) was assigned as the "index 
date" for cases and their matched controls. The pre-analysis lead-in period was defined as the 12 month period from $01 / \mathrm{Jan} / 2008$ to 31/Dec/2008, inclusive. Thus, the study inclusion period contains all time from 01/Jan/2008 to 31/Dec/2009, inclusive.

A base population was defined containing all patients eligible for this study. This was the denominator for calculations of the population prevalence of epilepsy. All cases and controls were selected from this population.

For inclusion in the base population, a patient must have been:

- registered on the database for the full lead-in period to allow the identification of existing epilepsy patients.

- $\quad$ registered on the database for the full analysis period to allow the characterisation of patients' current medication and comorbidities.

- $\quad$ aged between 0 and 120 years inclusive, on the index date

For the IHCIS, the analyses of medication use were restricted to those cases with full pharmacy benefits throughout the analysis period and no gaps in pharmacy benefit greater than one day during the analysis period.

\subsubsection{Analysis 1: prevalence of epilepsy}

The prevalence of epilepsy on the index date was determined, by gender and age category, as appropriate. The denominator was taken as all patients within the base population. In IHCIS, the numerator was calculated as all patients (within the denominator population) with two or more records of epilepsy, and 30 days or more between the first and last record of epilepsy. In GPRD, the numerator was calculated as all patients (within the denominator population) with an epilepsy diagnosis prior to the index date, and at least 2 anti-epileptic drug (AED) prescriptions within -3 and +6 months of any epilepsy diagnosis code AND at least 1 AED prescription within the lead-in or analysis period. For both databases, at least one of the diagnoses must be on, or before, the index date.

\subsubsection{Analysis 2: listing of comorbidities in epilepsy patients}

The prevalence of comorbidities within the epilepsy and control populations was determined. A patient was considered to have a comorbidity if they had one or more records of this condition within the time period being considered. Prevalence of comorbid disease in the epilepsy population was calculated as the number of epilepsy cases with a record of the disease (within the time periods defined below) divided by the total number of epilepsy cases. Prevalence of comorbid diseases in the controls were similarly calculated. Analyses were repeated for two time periods, (1) the analysis period and (2) the analysis period, and any time before, within the period of continuous enrollment.

In GPRD, the comorbidities are listed as defined by disease headings which were based on the Meddra Dictionary and were reviewed by clinicians. A Read/MedDRA (version 13.0) mapping was created using the available Read/MedDRA (version 6) dictionary and the 2010-AB release of the Unified Medical Language System (UMLS). Approximately $41 \%$ of events were not mapped, but many of the events remaining unmapped were not of clinical significance. Some of these were mapped by further review of terms.

In IHCIS, comorbidities are reported by Clinical Classifications Software (CCS) level 4 (HCUP, 2011). 
In addition, there were a number of conditions previously associated with epilepsy that were specifically explored including: depression, anxiety, bipolar disorder, suicidality, and migraine. These conditions were defined by clinician-reviewed coding lists using GPRD GOLD READ medical codes or ICD-9 codes for IHCIS.

In GPRD, medications are listed as defined by British National formulary (BNF) class, and in IHCIS by Universal System Classification (USC) Fourth Level. The USC is a categorization system, developed by IMS, to resolve a need for therapeutic classification of pharmaceutical products; the USC is widely accepted in North America as the standard for pharmaceutical product classification.

\subsubsection{Analysis 3: comparative analyses of the occurrence co-morbidities}

The relative prevalence of co-morbidities in epilepsy cases and matched controls was assessed. Unstratified matched analyses were conducted via conditional logistic regression using the SAS Proc PHREG procedure. Stratified analyses were conducted via the Fisher Exact tests using the SAS Proc FREQ procedure. Odds ratios (ORs) and 95\% confidence intervals are presented (Note: p-values are not presented). Since these were exploratory (rather than hypothesis testing) analyses, and sample sizes are not based on statistical considerations, there was no adjustment for multiple testing. ORs are only presented where there were at least five patients in both the case and control populations with a record of the comorbidity within the time period being considered. Results are presented for the 25 most frequent comorbidities amongst cases, and for the 25 highest ORs comparing cases and controls.

\subsubsection{Analysis 4: listing of prescribed medications used by epilepsy cases during a one-year period of observation}

The prevalence of drug use within the analysis period is presented for the epilepsy population. A patient was considered to be using the drug of interest if they were issued a prescription during the analysis period. The prevalence of drug use was calculated as the number of eligible cases with a record of use within the analysis period divided by the total number of eligible cases. Results are presented for cases only, and reported at Universal System Classification Fourth Level.

\section{Results}

There were 27,328 active epilepsy cases and 27,328 matched controls identified in the UK GPRD, and 83,045 active epilepsy cases and 83,045 controls identified from the IHCIS. Active epilepsy in GPRD was defined if a patient diagnosed with epilepsy had at least one AED prescription within the one-year lead-in or one-year analysis period. Active epilepsy in the IHCIS was defined if the patient had at least one diagnosis code within the lead-in or analysis period. The overall prevalence of active epilepsy was $0.8 \%$ in GPRD and $0.5 \%$ in IHCIS. The prevalence in GPRD increased with age and was highest in the age group 65 years and older. The prevalence was similar across the age groups in IHCIS with a peak of $0.5 \%$ in the age group 45 to 64 years.

The studies within the US claims database (IHCIS) and UK general practice database (GPRD) compared the prevalence of a range of psychiatric conditions including anxiety, 
bipolar disorder, depression, schizophrenia/psychosis and suicidality (Table 1\&Table 2). These psychiatric conditions were significantly more common in epilepsy cases than in controls (OR > 1.0, and lower bound of 95\% CI excluding 1.0). The highest ORs were for suicidality, bipolar disorder and schizophrenia. Migraine was also included in the analyses, and it was significantly more common among cases than controls for the time period during or before analysis period. However, the association was not significant for the analysis period only in GPRD, and the associations were stronger in IHCIS than GPRD. Given the chronic nature of these conditions the prevalence was considered both during and prior to the analysis period (i.e. the latter period represented the patients' medical histories).

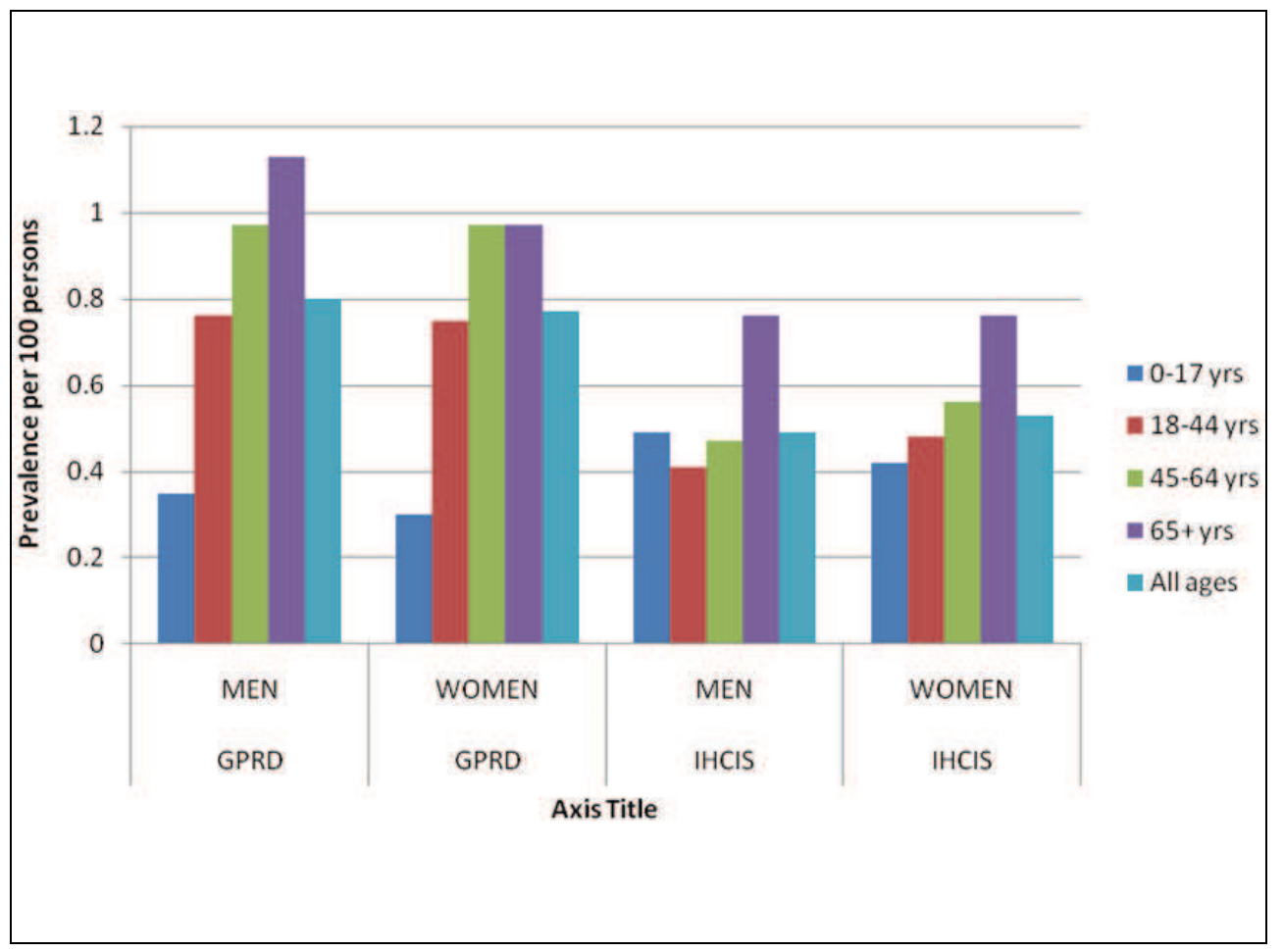

Fig. 1. The prevalence of active epilepsy per 100 persons on $1^{\text {st }}$ January 2009 in the GPRD and IHCIS databases, stratified by age and sex

The top 25 most common diagnosed comorbidities among cases are reported for within the analysis period (Table $3 \&$ Table 7) and within the analysis period or before (Table 4 \& Table 8), for GPRD and IHCIS respectively. As expected, the medical condition headings related to epilepsy, seizures and convulsions were at the top of the lists. Other conditions included respiratory infections, skin conditions, and hypertension. The latter conditions were also common in controls. 
The top 25 diagnosed comorbidities with the highest ORs comparing cases to controls were reported for the analysis period (Table $5 \&$ Table 9) and within the analysis period or before (Table 6 \& Table 10), for GPRD and IHCIS respectively. These listings can be considered for generating hypotheses about the associations between epilepsy and other conditions, though temporality cannot be established.

In the GPRD, mental retardation had a high OR of 24.9 (95\% CI: 12.3-50.4) during the analysis period. During the GPRD analysis period, the conditions with the highest ORs included many neurological conditions and behaviour and socialisation disturbances (Table 5). In the GPRD analysis period or ever before, the conditions with the highest ORs also included neurological and developmental conditions, and the highest ORs were 50.7 (95\% CI: 28.0-92.1) for "Cerebral disorders congenital", 38.1 (95\%CI: 20.9-69.3) for "Congenital and peripartum cerebral disorders", and 33.9 (95\%CI: 16.8-68.4) for "Neonatal neurological system disorders NEC" (Table 6).

The highest OR in the IHCIS was for intellectual disabilities, 143.1 (95\%CI: 84.6-241.9) during the analysis period, and 79.8 (95\% CI: 60.1-106.1) during the analysis period or ever before. The other conditions with high ORs included neurological disorders, cerebrovascular disease, developmental and learning disorders, and mental disorders (Table 9 \& Table 10).

Medication use was reported for the analysis year, 2009, for epilepsy cases only ( $N=27,328$ GPRD; N= 46,912 IHCIS). For IHCIS, only individuals with full pharmacy benefits throughout the year 2009 were included in medication analyses. The proportion of patients using anti-depressants and anti-epileptic drugs were reported using clinician-reviewed drug coding lists (Table 11 \& Table 13). In the GPRD, 97\% of the epilepsy cases had a prescription for an anti-epileptic drug in the analysis period, as expected since AED use either in the lead-in or analysis period was a requirement of the case definition ( $3 \%$ of cases only had AED use in the lead-in period). Eighteen percent of cases had a prescription for antidepressants in the one-year analysis period. In the IHCIS, $76 \%$ of epilepsy patients had a prescription for an AED, and $22 \%$ for anti-depressants in 2009. The $24 \%$ of epilepsy cases who did not have an AED in the analysis period may either not have had pharmacy benefit, or they were not prescribed an AED in that time period. However, the relative proportions of patients using each type of AED are of interest, and there are differences in the AED use between the UK GPRD and US IHCIS. Two of the older AEDs, valproate (34\%) and carbamazepine (32\%) were the most frequently used AEDs among epilepsy patients in the GPRD, followed by lamotrigine (18\%) and phenytoin $(17 \%)$. Lamotrigine $(15 \%)$ and phenytoin $(15 \%)$ were the most commonly used AEDs in the IHCIS in 2009, followed by carbamazepine $(12 \%)$ and topiramate $(9 \%)$.

The top 25 most commonly prescribed medication categories in a one year time period, 2009, are reported for GPRD (Table 12) and IHCIS (Table 14). As in (Table 11), drugs classified as "Control of Epilepsy" were the most commonly prescribed in GPRD (97.3\%). Other common medications were neuropathic pain $(52.3 \%)$, non-opioid analgesics $(39.7 \%)$, anti-manic drugs $(32.4 \%)$, non-steroidal anti-inflammatory drugs $(30.2 \%)$, opioid analgesics $(23.8 \%)$, broad spectrum penicillins (21.1\%), and statins $(20.2 \%)$. In the IHCIS, $74 \%$ of epilepsy patients had a prescription in the "Seizure disorders" drug category during 2009. The other frequently prescribed medications were codeine (23\%), extended spectrum macroli (19\%), benzodiazepines (17\%), and selective serotonin reuptake inhibitors (SSRI, 15\%). 


\begin{tabular}{|c|c|c|c|c|c|c|c|}
\hline \multirow[b]{2}{*}{ Comorbidity } & \multicolumn{5}{|l|}{ GPRD } & \multicolumn{2}{|l|}{ IHCIS } \\
\hline & $\begin{array}{c}\text { Number } \\
\text { Cases }\end{array}$ & $\begin{array}{c}\text { Percent } \\
\text { Cases }\end{array}$ & $\begin{array}{l}\text { Number } \\
\text { Controls }\end{array}$ & $\begin{array}{c}\text { Percent } \\
\text { Controls }\end{array}$ & $\begin{array}{c}\text { Odds Ratio } \\
(95 \% \text { CI })\end{array}$ & $\begin{array}{c}\text { Number } \\
\text { Cases }\end{array}$ & $\begin{array}{c}\text { Percent } \\
\text { Cases }\end{array}$ \\
\hline Anxiety & 750 & 2.74 & 677 & 2.48 & $1.1(1,1.2)$ & 9354 & 11.26 \\
\hline Bipolar Disorder & 26 & 0.1 & 7 & 0.03 & $3.7(1.6,8.6)$ & 2029 & 2.4 \\
\hline Depression & 1207 & 4.42 & 1034 & 3.78 & $1.2(1.1,1.3)$ & 9696 & 11 \\
\hline Migraine & 188 & 0.69 & 173 & 0.63 & $1.1(0.9,1.3)$ & 5806 & 6.99 \\
\hline $\begin{array}{l}\text { Schizophrenia } \\
\text { (GPRD)/ } \\
\text { Psychosis } \\
\text { (IHCIS) }\end{array}$ & 20 & 0.07 & 5 & 0.02 & $4.0(1.5,10.7)$ & 2215 & \\
\hline Suicidality & 76 & 0.28 & 20 & 0.07 & $3.8(2.3,6.2)$ & 1389 & 1.67 \\
\hline
\end{tabular}




\begin{tabular}{|c|c|c|c|c|c|c|c|}
\hline \multirow[b]{2}{*}{ Comorbidity } & \multicolumn{5}{|l|}{ GPRD } & \multicolumn{2}{|l|}{ IHCIS } \\
\hline & $\begin{array}{c}\text { Number } \\
\text { Cases }\end{array}$ & $\begin{array}{c}\text { Percent } \\
\text { Cases }\end{array}$ & $\begin{array}{l}\text { Number } \\
\text { Controls }\end{array}$ & $\begin{array}{l}\text { Percent } \\
\text { Controls }\end{array}$ & $\begin{array}{c}\text { Odds Ratio } \\
(95 \% \text { CI })\end{array}$ & $\begin{array}{c}\text { Number } \\
\text { Cases }\end{array}$ & $\begin{array}{c}\text { Percent } \\
\text { Cases }\end{array}$ \\
\hline Anxiety & 5858 & 21.44 & 4544 & 16.63 & $1.4(1.3,1.5)$ & 21214 & 25.55 \\
\hline $\begin{array}{l}\text { Bipolar } \\
\text { Disorder }\end{array}$ & 217 & 0.79 & 97 & 0.35 & $2.3(1.8,2.9)$ & 3829 & 4.61 \\
\hline Depression & 7988 & 29.23 & 6196 & 22.67 & $1.5(1.4,1.5)$ & 19762 & 23.8 \\
\hline Migraine & 2378 & 8.70 & 1841 & 6.74 & $1.3(1.3,1.4)$ & 13557 & 16.32 \\
\hline $\begin{array}{l}\text { Schizophrenia } \\
\text { (GPRD)/ } \\
\text { Psychosis } \\
\text { (IHCIS) }\end{array}$ & 299 & 1.09 & 108 & 0.40 & $2.8(2.3,3.6)$ & 7299 & 8.79 \\
\hline Suicidality & 1011 & 3.70 & 378 & 1.38 & $2.8(2.5,3.1)$ & 4877 & 5.87 \\
\hline
\end{tabular}




\begin{tabular}{|l|l|l|l|l}
\hline Comorbidity & $\begin{array}{l}\text { Number } \\
\text { Cases }\end{array}$ & $\begin{array}{l}\text { Percent } \\
\text { Cases }\end{array}$ & $\begin{array}{l}\text { Number } \\
\text { Controls }\end{array}$ \\
\hline Social issues & 4276 & 15.65 & 3527 & 1 \\
\hline Musculoskeletal and connective tissue pain and discomfort & 3751 & 13.73 & 3196 & 1 \\
\hline Coughing and associated symptoms & 3607 & 13.20 & 2728 & 9 \\
\hline Upper respiratory tract infections NEC & 3112 & 11.39 & 2693 & 9 \\
\hline Upper respiratory tract infections & 3111 & 11.38 & 2693 & 9 \\
\hline Joint related signs and symptoms & 2826 & 10.34 & 2405 & 8 \\
\hline Seizures and seizure disorders NEC & 2638 & 9.65 & 11 & 0 \\
\hline Dietary and nutritional therapies & 2441 & 8.93 & 1977 & 7 \\
\hline General signs and symptoms NEC & 2313 & 8.46 & 1482 & 5 \\
\hline Reproductive organ and breast histopathology procedures & 2262 & 8.28 & 2499 & 9 \\
\hline Lower respiratory tract and lung infections & 1946 & 7.12 & 1138 & 4 \\
\hline Lower respiratory tract infections NEC & 1936 & 7.08 & 1125 & 4 \\
\hline Non-site specific injuries NEC & 1548 & 5.66 & 876 & 3 \\
\hline Pain and discomfort NEC & 1450 & 5.31 & 1116 & 4 \\
\hline Bronchospasm and obstruction & 1444 & 5.28 & 1128 & 4 \\
\hline Neurological signs and symptoms NEC & 1429 & 5.23 & 764 & 2 \\
\hline Dermatitis and eczema & 1400 & 5.12 & 1076 & 3 \\
\hline Urinary tract infections & 1349 & 4.94 & 754 & 2 \\
\hline Asthenic conditions & 1343 & 4.91 & 862 & 3 \\
\hline Gastrointestinal and abdominal pains (excl oral and throat) & 1318 & 4.82 & 1092 & 4 \\
\hline Vascular hypertensive disorders NEC & 1207 & 4.42 & 1381 & 5 \\
\hline Oral soft tissue infections & 1174 & 4.30 & 973 & 3 \\
\hline Bladder and urethral symptoms & 1135 & 4.15 & 683 & 2 \\
\hline Gastrointestinal atonic and hypomotility disorders NEC & 1128 & 4.13 & 598 & 2 \\
\hline Headaches NEC & 1079 & 3.95 & 751 & 2 \\
\hline
\end{tabular}




\begin{tabular}{|c|c|c|c|c|}
\hline Comorbidity & $\begin{array}{l}\text { Number } \\
\text { Cases }\end{array}$ & $\begin{array}{l}\text { Percent } \\
\text { Cases } \\
\end{array}$ & $\begin{array}{l}\text { Number } \\
\text { Controls }\end{array}$ & $\begin{array}{l}\text { Percent } \\
\text { Control }\end{array}$ \\
\hline Seizures and seizure disorders NEC & 26225 & 95.96 & 279 & 1.02 \\
\hline Social issues & 17790 & 65.10 & 16830 & 61.59 \\
\hline Upper respiratory tract infections NEC & 16529 & 60.48 & 15504 & 56.73 \\
\hline Upper respiratory tract infections & 16529 & 60.48 & 15504 & 56.73 \\
\hline $\begin{array}{l}\text { Musculoskeletal and connective tissue pain and } \\
\text { discomfort }\end{array}$ & 15299 & 55.98 & 14339 & 52.47 \\
\hline Coughing and associated symptoms & 13657 & 49.97 & 11438 & 41.85 \\
\hline General signs and symptoms NEC & 12478 & 45.66 & 8900 & 32.57 \\
\hline Joint related signs and symptoms & 12153 & 44.47 & 10691 & 39.12 \\
\hline Dietary and nutritional therapies & 11088 & 40.57 & 10941 & 40.04 \\
\hline Non-site specific injuries NEC & 10949 & 40.07 & 7931 & 29.02 \\
\hline Dermatitis and eczema & 10442 & 38.21 & 8736 & 31.97 \\
\hline Lower respiratory tract and lung infections & 10357 & 37.90 & 7734 & 28.30 \\
\hline Lower respiratory tract infections NEC & 10326 & 37.79 & 7709 & 28.21 \\
\hline $\begin{array}{l}\text { Reproductive organ and breast histopathology } \\
\text { procedures }\end{array}$ & 9986 & 36.54 & 10502 & 38.43 \\
\hline Pain and discomfort NEC & 8849 & 32.38 & 7585 & 27.76 \\
\hline Oral soft tissue infections & 8693 & 31.81 & 8108 & 29.67 \\
\hline $\begin{array}{l}\text { Gastrointestinal and abdominal pains (excl oral } \\
\text { and throat) }\end{array}$ & 8637 & 31.60 & 7805 & 28.56 \\
\hline Asthenic conditions & 8219 & 30.08 & 5825 & 21.32 \\
\hline Neurological signs and symptoms NEC & 7933 & 29.03 & 4404 & 16.12 \\
\hline Skin structures and soft tissue infections & 7823 & 28.63 & 5654 & 20.69 \\
\hline Headaches NEC & 7733 & 28.30 & 5677 & 20.77 \\
\hline Cardiac signs and symptoms NEC & 7544 & 27.61 & 5307 & 19.42 \\
\hline $\begin{array}{l}\text { Allergies to foods, food additives, drugs and } \\
\text { other chemicals }\end{array}$ & 7499 & 27.44 & 6002 & 21.96 \\
\hline Depressive disorders & 7177 & 26.26 & 5660 & 20.71 \\
\hline Urinary tract infections & 7098 & 25.97 & 5133 & 18.78 \\
\hline
\end{tabular}


Seizures and seizure disorders NEC

\section{Cases}

Mental retardations

Withdrawal and rebound effects

Disability issues

Abnormal behaviour NEC

Sodium imbalance

Pervasive developmental disorders NEC

Behaviour and socialisation disturbances

Calcium metabolism disorders

Cerebellar coordination and balance disturbances

Therapeutic bladder catheterisation

Lower respiratory tract inflammatory and

immunologic conditions

Nervous system therapeutic procedures NEC

Salivary gland disorders NEC

Confusion and disorientation

Cortical dysfunction NEC

Thoracic cage fractures non-spinal (excl pathological)

Thoracic cage fractures and dislocations

Schizophrenia NEC

Bipolar disorders

Psychiatric elimination disorders

Tremor (excl congenital)

Nervous system haemorrhagic disorders

Delusional symptoms

Drug withdrawal therapies

277

47

156

44
197

35

179

199

32

32
22

22

26

42

223

31

23

33

\begin{tabular}{|l|l}
\hline 2638 & 9. \\
\hline 199 & 0.7 \\
46 & 0.
\end{tabular}

Cases Controls

9.65

0.73

0.17

\begin{tabular}{|l|l}
\hline 50 & 0.18 \\
\hline
\end{tabular}

109

\begin{tabular}{l|l}
35 & 0.13
\end{tabular}

\begin{tabular}{l|l}
66 & 0.24 \\
\hline
\end{tabular}

\begin{tabular}{|l|l}
66 & 0.2 \\
\hline 49 & 0.1 \\
\hline 179 & 0.6 \\
\hline
\end{tabular}

\begin{tabular}{l|l}
\hline 79 & 0.66 \\
\hline 99
\end{tabular}

\begin{tabular}{l|l|l}
\hline 0.18 & 12 & 0.0 \\
\hline 0.66 & 9 & 0.0
\end{tabular}

\begin{tabular}{|l|l|l}
\hline .18 & 9 & 0.03 \\
\hline & 0.06 & 38
\end{tabular}

\begin{tabular}{l|l|l}
\hline 79 & 0.66 & 38 \\
\hline 99 & 0.73 & 43 \\
\hline 2 & 0.12 & 7 \\
\hline
\end{tabular}

0.12

\begin{tabular}{l|l|l}
0.12 & 7 & 0.03 \\
\hline 0.12 & 7 & 0.02
\end{tabular}

\begin{tabular}{|l|l|l}
\hline 0.08 & 5 & 0.02 \\
\hline 0.10 & 6 & 0.02 \\
\hline
\end{tabular}

\begin{tabular}{|l|l|l}
\hline 0.10 & 6 & 0.02 \\
\hline 0.15 & 10 & 0.02 \\
\hline 0.82 & 57 & 0.27 \\
\hline 0.11 & 8 & 0.02
\end{tabular}

0.11

0.11

0.12

\begin{tabular}{|l|l}
\hline 57 & 0.21 \\
\hline 8 & 0.03 \\
\hline 6 & 0.02 \\
\hline 9 & 0.03 \\
\hline
\end{tabular}




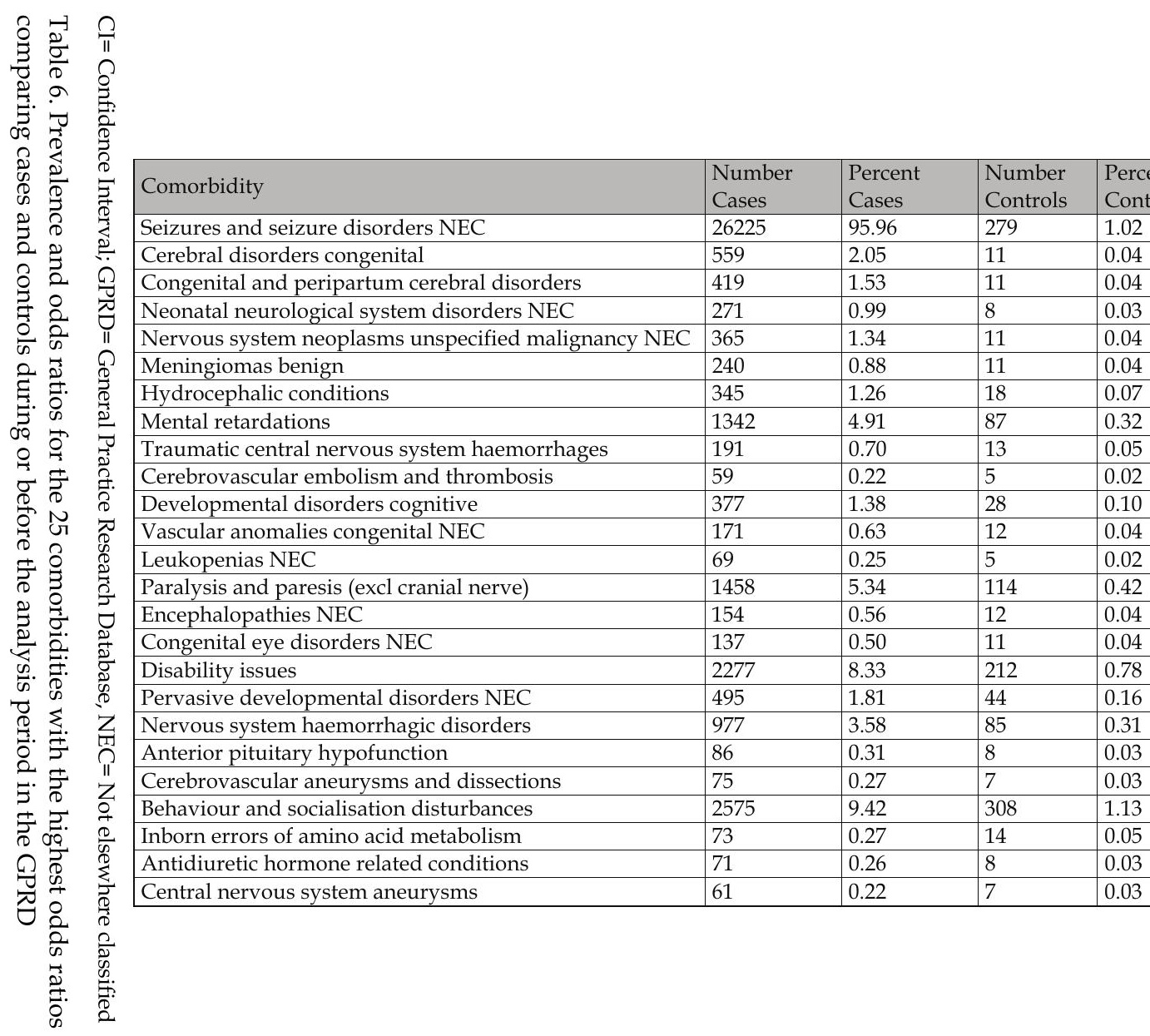


Other skin disorders

Other and unspecified upper respiratory infections

Other nervous system symptoms and disorders

Malaise and fatigue

Other upper respiratory disease

45635

25108

24367

23827

23526

204

20413

Abdominal pain

Other eye disorders

Other injuries and conditions due to external causes

Depressive disorders

Other central nervous system disorders

Nonspecific chest pain

Acute upper respiratory infections of multiple or unspecified sites Other headache

Other and unspecified gastrointestinal disorders

Other bone disease and musculoskeletal deformities

Other thyroid disorders

18437

18437

18

17051

15474

13586

12762

113

11335

11228
10907

\begin{tabular}{l|l}
10926 \\
10769
\end{tabular}

1072

\begin{tabular}{|l|l|}
\hline 10722 \\
\hline 10462 \\
\hline 1012 \\
\hline 9909 \\
\hline 9886 \\
\hline
\end{tabular}

\begin{tabular}{l|l|l|l}
\hline $\begin{array}{l}\text { Percent } \\
\text { Cases }\end{array}$ & $\begin{array}{l}\text { Number } \\
\text { Controls }\end{array}$ & $\begin{array}{l}\text { Perc } \\
\text { Con }\end{array}$ \\
\hline 54.95 & 0 & 0 \\
\hline 49.46 & 9 & 0.01 \\
\hline 30.23 & 16519 & 19.8 \\
\hline 29.34 & 19961 & 24.0 \\
\hline 28.69 & 20398 & 24.5 \\
\hline 28.33 & 21045 & 25.3 \\
\hline 24.58 & 12854 & 15.4 \\
\hline 24.40 & 9946 & 11.9 \\
\hline 22.20 & 12774 & 15.3 \\
\hline 21.76 & 14957 & 18.0 \\
\hline 20.53 & 14692 & 17.6 \\
\hline 18.63 & 4413 & 5.31 \\
\hline 16.36 & 7983 & 9.61 \\
\hline 15.37 & 9794 & 11.7 \\
\hline 14.34 & 7637 & 9.20 \\
\hline 13.65 & 8142 & 9.80 \\
\hline 13.52 & 4945 & 5.95 \\
\hline 13.13 & 4801 & 5.78 \\
\hline 13.04 & 2403 & 2.89 \\
\hline 12.97 & 6747 & 8.12 \\
\hline 12.91 & 8206 & 9.88 \\
\hline 12.60 & 4132 & 4.98 \\
\hline 12.19 & 5516 & 6.64 \\
\hline 11.93 & 7073 & 8.52 \\
\hline 11.90 & 6979 & 8.40 \\
\hline & & \\
\hline
\end{tabular}




\begin{tabular}{|l|l|l|l|l|}
\hline Comorbidity & $\begin{array}{l}\text { Number } \\
\text { Cases }\end{array}$ & $\begin{array}{l}\text { Percent } \\
\text { Cases }\end{array}$ & $\begin{array}{l}\text { Number } \\
\text { Controls }\end{array}$ & $\begin{array}{l}\text { Percent } \\
\text { Controls }\end{array}$ \\
\hline Convulsions & 76929 & 92.64 & 58 & 0.07 \\
\hline Epilepsy & 69782 & 84.03 & 0 & 0 \\
\hline Other connective tissue disease & 50298 & 60.57 & 36898 & 44.43 \\
\hline Other and unspecified lower respiratory disease & 46270 & 55.72 & 31447 & 37.87 \\
\hline Other non-traumatic joint disorders & 42216 & 50.84 & 31641 & 38.10 \\
\hline Other skin disorders & 41651 & 50.15 & 34760 & 41.86 \\
\hline Other and unspecified upper respiratory infections & 41109 & 49.50 & 36734 & 44.23 \\
\hline Other nervous system symptoms and disorders & 37912 & 45.65 & 11592 & 13.96 \\
\hline Disorders of lipid metabolism & 37001 & 44.56 & 32157 & 38.72 \\
\hline Malaise and fatigue & 35255 & 42.45 & 21419 & 25.79 \\
\hline Essential hypertension & 33858 & 40.77 & 27249 & 32.81 \\
\hline Other upper respiratory disease & 33727 & 40.61 & 26344 & 31.72 \\
\hline Abdominal pain & 32829 & 39.53 & 23175 & 27.91 \\
\hline Other injuries and conditions due to external causes & 32800 & 39.50 & 16637 & 20.03 \\
\hline Nonspecific chest pain & 31341 & 37.74 & 20177 & 24.30 \\
\hline $\begin{array}{l}\text { Acute upper respiratory infections of multiple or } \\
\text { unspecified sites }\end{array}$ & 30716 & 36.99 & 24805 & 29.87 \\
\hline Other headache & 30126 & 36.28 & 13070 & 15.74 \\
\hline Allergic reactions & 28909 & 34.81 & 22971 & 27.66 \\
\hline Other eye disorders & 28567 & 34.40 & 21219 & 25.55 \\
\hline Other and unspecified gastrointestinal disorders & 27635 & 33.28 & 17061 & 20.54 \\
\hline Superficial injury; contusion & 26869 & 32.35 & 15552 & 18.73 \\
\hline Other ear and sense organ disorders & 26352 & 31.73 & 18559 & 22.35 \\
\hline Other and unspecified circulatory disease & 25911 & 31.20 & 15087 & 18.17 \\
\hline Other central nervous system disorders & 25633 & 30.87 & 4591 & 5.53 \\
\hline Sprains and strains & 25339 & 30.51 & 20144 & 24.26 \\
\hline & & & & \\
\hline
\end{tabular}




\begin{tabular}{|l|l|l|l|l|l}
\hline Comorbidity & $\begin{array}{l}\text { Number } \\
\text { Cases }\end{array}$ & $\begin{array}{l}\text { Percent } \\
\text { Cases }\end{array}$ & $\begin{array}{l}\text { Number } \\
\text { Controls }\end{array}$ & $\begin{array}{l}\text { Percent } \\
\text { Controls }\end{array}$ \\
\hline Convulsions & 41078 & 49.46 & 9 & 0.01 & 1 \\
\hline Intellectual disabilities & 2010 & 2.42 & 17 & 0.02 & 1 \\
\hline Cancer of brain and nervous system & 1705 & 2.05 & 43 & 0.05 & 3 \\
\hline Developmental disabilities & 1312 & 1.58 & 45 & 0.05 & 3 \\
\hline Other paralysis & 3942 & 4.75 & 116 & 0.14 & 3 \\
\hline Congenital hip dislocation & 217 & 0.26 & 9 & 0.01 & 2 \\
\hline Other nervous system congenital anomalies & 864 & 1.04 & 34 & 0.04 & 2 \\
\hline Learning disorders & 1792 & 2.16 & 88 & 0.11 & 2 \\
\hline Cerebrovascular anomalies & 431 & 0.52 & 20 & 0.02 & 2 \\
\hline Hemiplegia & 1724 & 2.08 & 92 & 0.11 & 1 \\
\hline Motor skill disorders & 304 & 0.37 & 17 & 0.02 & 1 \\
\hline Encephalitis (except that caused by TB or STD) & 340 & 0.41 & 19 & 0.02 & 1 \\
\hline Benign neoplasm of cerebral meninges & 712 & 0.86 & 43 & 0.05 & 1 \\
\hline Pervasive developmental disorders & 1979 & 2.38 & 140 & 0.17 & 1 \\
\hline Intrauterine hypoxia and birth asphyxia & 72 & 0.09 & 5 & 0.01 & 1 \\
\hline Intracranial hemorrhage & 1237 & 1.49 & 92 & 0.11 & 1 \\
\hline Respiratory distress syndrome & 65 & 0.08 & 5 & 0.01 & 1 \\
\hline Congenital anomalies of skull and facial bones & 150 & 0.18 & 13 & 0.02 & 1 \\
\hline Aspiration pneumonitis; food/vomitus & 952 & 1.15 & 85 & 0.10 & 1 \\
\hline Spina bifida & 215 & 0.26 & 20 & 0.02 & 1 \\
\hline Congenital hip deformity & 211 & 0.25 & 20 & 0.02 & 1 \\
\hline Occlusion of cerebral arteries & 4102 & 4.94 & 456 & 0.55 & $\subseteq$ \\
\hline Late effects of cerebrovascular disease & 3354 & 4.04 & 375 & 0.45 & $\subseteq$ \\
\hline Other dependence on machines & 59 & 0.07 & 6 & 0.01 & $\subseteq$ \\
\hline Acute but ill-defined cerebrovascular accident & 3526 & 4.25 & 437 & 0.53 & 5 \\
\hline
\end{tabular}




\begin{tabular}{|l|l|l|l|l|}
\hline Comorbidity & $\begin{array}{l}\text { Number } \\
\text { Cases }\end{array}$ & $\begin{array}{l}\text { Percent } \\
\text { Cases }\end{array}$ & $\begin{array}{l}\text { Number } \\
\text { Controls }\end{array}$ & $\begin{array}{l}\text { Percent } \\
\text { Control }\end{array}$ \\
\hline Convulsions & 76929 & 92.64 & 58 & 0.07 \\
\hline Intellectual disabilities & 3836 & 4.62 & 52 & 0.06 \\
\hline Microcephalus & 818 & 0.99 & 12 & 0.01 \\
\hline Congenital hydrocephalus & 780 & 0.94 & 17 & 0.02 \\
\hline Cancer of brain and nervous system & 3219 & 3.88 & 121 & 0.15 \\
\hline Developmental disabilities & 3129 & 3.77 & 153 & 0.18 \\
\hline Other nervous system congenital anomalies & 2545 & 3.06 & 119 & 0.14 \\
\hline Other paralysis & 6590 & 7.94 & 315 & 0.38 \\
\hline Hemiplegia & 4776 & 5.75 & 285 & 0.34 \\
\hline Cerebrovascular anomalies & 1506 & 1.81 & 87 & 0.10 \\
\hline Intracranial hemorrhage & 4660 & 5.61 & 307 & 0.37 \\
\hline Learning disorders & 4539 & 5.47 & 324 & 0.39 \\
\hline Benign neoplasm of cerebral meninges & 1530 & 1.84 & 98 & 0.12 \\
\hline Coma; stupor; and brain damage & 20114 & 24.22 & 1715 & 2.07 \\
\hline Encephalitis (except that caused by TB or STD) & 1264 & 1.52 & 80 & 0.10 \\
\hline Motor skill disorders & 1047 & 1.26 & 79 & 0.10 \\
\hline Pervasive developmental disorders & 2978 & 3.59 & 251 & 0.30 \\
\hline Aspiration pneumonitis; food/vomitus & 2675 & 3.22 & 218 & 0.26 \\
\hline Occlusion of cerebral arteries & 11582 & 13.95 & 1286 & 1.55 \\
\hline Secondary malignancy of brain/spine & 619 & 0.75 & 52 & 0.06 \\
\hline Congenital hip dislocation & 569 & 0.69 & 49 & 0.06 \\
\hline $\begin{array}{l}\text { Mental disorders due to general medical } \\
\text { conditions not elsewhere classified }\end{array}$ & 2139 & 2.58 & 192 & 0.23 \\
\hline Dissociative disorders & 185 & 0.22 & 16 & 0.02 \\
\hline Acute but ill-defined cerebrovascular accident & 9617 & 11.58 & 1233 & 1.48 \\
\hline Late effects of cerebrovascular disease & 7766 & 9.35 & 967 & 1.16 \\
\hline & & & & \\
\hline
\end{tabular}




\begin{tabular}{|l|r|}
\hline Drug & N (\%) \\
\hline Anti-Epileptic Drugs & $26584(97.3)$ \\
\hline Anti-depressants & $4806(17.6)$ \\
\hline Valproic acid & $9293(34.0)$ \\
\hline Carbamazepine & $8776(32.1)$ \\
\hline Lamotrigine & $5020(18.4)$ \\
\hline Phenytoin & $4757(17.4)$ \\
\hline Levetiracetam & $2977(10.9)$ \\
\hline Phenobarbital & $1645(6.02)$ \\
\hline Clobazam & $1161(4.3)$ \\
\hline Topiramate & $1133(4.2)$ \\
\hline Gabapentin & $784(2.9)$ \\
\hline Clonazepam & $733(2.7)$ \\
\hline Primidone & $570(2.1)$ \\
\hline Pregabalin & $507(1.9)$ \\
\hline
\end{tabular}

GPRD = General Practice Research Database. Note: the following anti-epileptic drugs were prescribed to $<1 \%$ of the patients with epilepsy: zonisamide, oxcarbazepine, ethosuximide, lacosamide, vigabitrin, acetazolamide, rufinamide, tiagabine hydrochloride, paraldehyde, stiripentol, sultiame or mesuximide. No patients were prescribed beclamide, dipotassium clorazepate, eslicarbazepine acetate, or fosphenytoin sodium.

Table 11. Prevalence of use of anti-convulsants and anti-depressants in epilepsy patients during the one-year period of observation from 01 Jan 2009 to 31 Dec 2009 in GPRD

\begin{tabular}{|l|r|}
\hline Drug Category & N (\%) \\
\hline Control of epilepsy & $26595(97.3)$ \\
\hline Neuropathic pain & $14297(52.3)$ \\
\hline Non-opioid analgesics & $10861(39.7)$ \\
\hline Antimanic drugs & $8859(32.4)$ \\
\hline Non-steroidal anti-inflammatory drugs & $8249(30.2)$ \\
\hline Opioid analgesics & $6516(23.8)$ \\
\hline Broad-spectrum penicillins & $5763(21.1)$ \\
\hline Statins & $5530(20.2)$ \\
\hline Urinary-tract infections & $5308(19.4)$ \\
\hline Proton pump inhibitors & $5244(19.2)$ \\
\hline Topical corticosteroids & $4856(17.8)$ \\
\hline Antiplatelet drugs & $4587(16.8)$ \\
\hline Selective beta-2-agonists & $3983(14.6)$ \\
\hline Anxiolytics & $3684(13.5)$ \\
\hline Emollient skin preparations & $3535(12.9)$ \\
\hline Osmotic laxatives & $3362(12.3)$ \\
\hline Hypnotics & $3345(12.2)$ \\
\hline Angiotensin-converting enzyme inhibitors & $3274(12.0)$ \\
\hline Skeletal muscle relaxants & $3064(11.2)$ \\
\hline Anxiolytics and neuroleptics (in anaesthesia) & $3041(11.1)$ \\
\hline Selective serotonin re-uptake inhibitors & $2886(10.6)$ \\
\hline Prophylaxis of migraine & $2863(10.5)$ \\
\hline Corticosteroids (inhaled for respiratory conditions) & $2859(10.5)$ \\
\hline Beta-adrenoceptor blocking drugs & $2819(10.3)$ \\
\hline Drugs used in nausea and vertigo & $2747(10.1)$ \\
\hline
\end{tabular}

$\mathrm{BNF}=$ British National Formulary $; \mathrm{GPRD}=$ General Practice Research Database

Table 12. Prevalence of use of the top 25 most frequent medications in epilepsy patients during the one-year period of observation from 01 Jan 2009 to 31 Dec 2009, by BNF Category in GPRD 


\begin{tabular}{|l|r|}
\hline Drug & $\mathbf{N ~ ( \% )}$ \\
\hline Anti-depressants & $10378(22.1)$ \\
\hline Anticonvulsants- antiepileptics & $35543(75.8)$ \\
\hline Lamotrigine & $7157(15.3)$ \\
\hline Phenytoin & $6877(14.7)$ \\
\hline Carbamazepine & $5549(11.8)$ \\
\hline Topiramate & $3976(8.5)$ \\
\hline Clonazepam & $2973(6.3)$ \\
\hline Gabapentin & $2279(4.9)$ \\
\hline Phenobarbital & $2070(4.4)$ \\
\hline Valproate & $503(1.1)$ \\
\hline Ethosuximide & $428(0.9)$ \\
\hline Methsuximide & $16(0.03)$ \\
\hline
\end{tabular}

IHCIS= Integrated Healthcare Information Services

Table 13. Prevalence of use of anti-convulsants and anti-depressants in epilepsy patients during the one-year period of observation from 01 Jan 2009 to 31 Dec 2009 in IHCIS

\begin{tabular}{|l|r|}
\hline Drug Category & $\mathbf{N}(\mathbf{\%})$ \\
\hline Seizure disorders & $34723(74.0)$ \\
\hline Codeine \& Comb, Non-Inj & $10742(22.9)$ \\
\hline Extended spectrum macroli & $8784(18.7)$ \\
\hline Benzodiazepines & $7715(16.5)$ \\
\hline SSRI & $7037(15.0)$ \\
\hline HMG-COA reductase inhibitors & $6693(14.3)$ \\
\hline Aminopenicillins & $6304(13.4)$ \\
\hline Hormones, Cort plain, oral & $5941(12.7)$ \\
\hline Cephalosporins \& related & $5829(12.4)$ \\
\hline Proton pump inhibitors & $5642(12.0)$ \\
\hline Anti-arthritis, plain & $5627(12.0)$ \\
\hline Quinolones, systemic & $5525(11.8)$ \\
\hline Beta blockers & $4332(9.2)$ \\
\hline Steroid, inhaled nasal & $4244(9.1)$ \\
\hline B-lactam, increased activity & $4212(9.0)$ \\
\hline Mus relx, non-surg,W/O ANA & $4150(8.9)$ \\
\hline Hormones,Cort plain, derm & $3732(8.0)$ \\
\hline Thyroid hormone, synth & $3616(7.7)$ \\
\hline ACE Inhib, alone & $3579(7.6)$ \\
\hline Beta agonists, aerosol & $3522(7.5)$ \\
\hline Antivirals, other & $3482(7.4)$ \\
\hline Sulfa \& Trimeth comb & $3020(6.4)$ \\
\hline Calcium blockers & $2690(5.7)$ \\
\hline Antipsychotics, other & $2638(5.6)$ \\
\hline Analeptics & $2493(5.3)$ \\
\hline
\end{tabular}

Table 14. Prevalence of use of the top 25 most frequent medications in epilepsy patients during the one-year period of observation from 01 Jan 2009 to 31 Dec 2009, by Universal System Classification Fourth Level in IHCIS 


\section{Discussion}

Electronic medical record systems provide an opportunity to identify treatment patterns and comorbidities in a large diagnosed disease population. They also allow for comparisons between the relative prevalence estimates of various comorbidities within the same data source. The comorbidities were ranked by how prevalent they were in epilepsy cases, and then by the ORs comparing cases to controls. The conditions with the highest ORs have the strongest associations with epilepsy compared to matched controls, therefore these findings may be hypothesis-generating for conditions related to epilepsy, though temporality cannot be established in this cross-sectional study. For instance, these can be tested as risk factors for epilepsy; conversely, epilepsy can be examined as a risk factor for these associated conditions.

The definition of both cases and controls requires that patients are registered throughout the full lead-in and analysis periods. The lead-in period is used to identify existing epilepsy patients to ensure that all cases have epilepsy prior to the start of the observation period. This ensures that patients are "at risk" of receiving management for epilepsy throughout the observation period when the patient's status is being assessed. This cross sectional approach, based on a set calendar index date, means there is no restriction of the duration of epilepsy prior to the start of observation, and the population is likely to contain both prevalent and newly-diagnosed epilepsy cases. The use of an index date tied to an individual patient (such as their first diagnosis on a database) is another possible approach, which is more tailored incident cases (or patients at an earlier stage of the disease) and historical rather than recent management patterns.

Diagnosed prevalence rates were similar in a US insurance claims database $(0.5 \%)$ and a UK general practice-based electronic medical record system $(0.8 \%)$, although peak age prevalence differed, likely based on the patient populations covered by these systems. The IHCIS system under-represents the elderly population, because it mainly consists of the employed, insured population. These prevalence estimates are similar to those reported in the literature for studies with records-based methodology, ranging from 3 to 8 per 1000 persons (Banerjee et al., 2009).

Many diagnosed comorbidities, including psychiatric and neurological comorbidities, were more prevalent in the patients with epilepsy compared to matched controls. A previous study in the GPRD also reported that many conditions were associated with epilepsy (Gaitatzis, 2004). The current study supports the evidence that the burden of epilepsy along with its comorbidities is high compared to the general population.

Major depression is a common co-morbidity of epilepsy with at least $20 \%$ of epilepsy patients experiencing mild to severe depressive symptoms ( $\mathrm{O}^{\prime}$ Donoghue et al., 1999; Mendez et al., 1986; Hermann et al., 1986; Baker et al., 1996; Cramer et al., 2004; Jacoby et al., 1996; Boylan et al., 2004; Beghi et al., 2002). In the current study, $29 \%$ of patients with epilepsy in the GPRD (OR=1.5, 95\% CI: 1.4-1.5), and $24 \%$ of patients with epilepsy in the IHCIS (OR=2.6, 95\%CI: 2.5-2.7) had a diagnosis of depression in the analysis period or before. Studies using structured psychiatric interviews in epilepsy clinics, likely to represent more severe epilepsy patients, have reported a prevalence of major depression of up to 55\% (Mendez et al., 1996). However, the cross-sectional nature of most studies prevents inferences concerning the temporal order of disease development. When 
longitudinal analyses have been completed a history of major depression has been associated with an increased risk for incident epilepsy and first unprovoked seizure (Nilsson et al., 2003; Forsgren \& Nystrom 1990; Hesdorffer et al., 2006; Hesdorffer et al., 2000).

Certain epilepsy types may be at increased risk of depression: the mean estimated lifetime prevalence of depression is $30 \%$ for patients with temporal lobe epilepsy and refractory epilepsy with corresponding risks in the general population of between $6 \%$ and $17 \%$ (Glosser et al., 2000). Studies have consistently identified co-morbid depression as a powerful predictor of poor health related quality of life in epilepsy patients even after adjustment for seizure frequency and severity (Kanner, 2009). Depression is also a strong predictor for increased suicidality among epilepsy patients (Kanner, 2009).

The prevalence of anxiety disorders is consistently higher (approximately twofold increased risk) among epilepsy patients than the general population. In the current study, $21 \%$ of patients with epilepsy in the GPRD (OR=1.4, 95\%CI: 1.3-1.5), and $26 \%$ of patients with epilepsy in the IHCIS (OR=2.1, 95\%CI: 2.1-2.2) had a diagnosis of anxiety in the analysis period or before. Studies using a range of disease measurement scales, and capturing patients mainly through cross sectional health surveys, have reported point prevalence estimates in epilepsy patients of $11 \%$ to 39\% (Gaitatzis et al., 2004; Tellez-Zenteno et al., 2007; Edeh \& Toone, 1987; Stirne et al., 2005; Kobau et al., 2006). The variation is likely to reflect the selection of different subsets of the epilepsy population with higher prevalence estimates observed among patients with temporal lobe epilepsy.

The prevalence of psychoses in epilepsy, as reported from population-based studies, ranges from $2 \%$ to $7 \%$ (Gaitatzis et al., 2004). The prevalence varies by epilepsy type with estimates of $10 \%$ to $19 \%$ reported among individuals with temporal lobe epilepsy, nearly double the risk associated with generalised epilepsy, though larger differences have been reported in older studies (Gaitatzis et al., 2004; Shukla et al., 1979). Gender differences have also been reported with females at an increased risk of psychoses. An Icelandic study reported 6\% prevalence in males with epilepsy versus $9 \%$ in females (Gudmundsson, 1966). In the current study, $9 \%$ of patients with epilepsy in the IHCIS (OR=9.7, 95\%CI: 9.0-10.4) had a diagnosis of psychosis in the analysis period or before.

The incidence of psychoses is reported to be 3 to 12 times higher in epilepsy patients than the general population. The range again depends on the epilepsy population sampled (seizure type and severity) as well as the instrument used to diagnose psychosis (Bredkjaer et al., 1998; Torta \& Keller, 1999). Patients undergoing epilepsy surgery are at particular risk of developing psychoses for the first time. Interictal psychoses can develop in $3 \%$ to $12 \%$ of patients following anterior temporal lobectomy and seizure-related psychoses can affect $1 \%$ to $13 \%$ of patients (Gaitatzis et al., 2004; Gaitatzis et al., 2004 (2)).

Studies concentrating solely on schizophrenia have reported prevalence estimates of $4 \%$ to $18 \%$ among patients with epilepsy (the majority suffering from temporal lobe epilepsy) (Gaitatzis et al., 2004; Gaitatzis et al., 2004 (2)). The expected prevalence of schizophrenia in the general population is $1 \%$ (Toone, 2000). Patients with schizophrenia are also at an increased risk of seizures related to the condition itself and to exposure to psychotropic medications that lower seizure threshold (e.g. clozapine) (Torta \& Keller, 1999). In the current study, $1 \%$ of patients with epilepsy in the GPRD (OR=2.8, 95\%CI: 2.5-3.1) had a diagnosis of schizophrenia in the analysis period or before. 
Suicide is a much discussed cause of death in epilepsy patients, especially with the recent analysis by the US Food and Drug Administration in 2008 that showed an association between AEDs and suicidality in pooled clinical trials. Population-based studies report that between $0 \%$ and 9\% (Sweden and Iceland, respectively) of deaths among patients with epilepsy result from suicide (Tomson et al., 2004). However it is not clear whether epilepsy or its treatment increases the risk of suicide: suicide attempt has been associated with an increased risk for developing epilepsy indicating the association could represent the recurrence, post epilepsy diagnosis, of an underlying suicidality risk 74. The GPRD and IHCIS results indicate an association between epilepsy diagnosis and suicidality, with an OR of 2.8 (95\% CI: 2.5-3.1) in GPRD and 5.5 (95\% CI: 5.2-6.0) in IHCIS for during or ever before the analysis period.

The reported prevalence of migraine among epilepsy patients ranges from $8 \%$ to $23 \%$ (Marks \& Ehrenberg, 1993) compared with 12\% in the general population (Lipton et al., 2007). While the majority of studies report the risk of migraine to be two-fold greater among epilepsy patients (Lipton et al., 1994), two studies failed to confirm this association (KaraaliSavrun et al., 2002; Nuyen et al., 2006). This discordance is most likely due to differences in migraine definition and the absence of well defined control groups in some studies. Recent studies indicate that the association is primarily with migraine with aura, though the absolute prevalence of migraine with aura among epilepsy patients has yet to be reported (Piccinelli et al., 2006).

The IHCIS and GPRD database studies supported a positive association between epilepsy and migraine. A history of diagnosed migraine was present in 16\% of epilepsy cases and $5 \%$ of controls in the IHCIS database study (OR $=3.7,95 \% \mathrm{CI}: 3.6-3.8)$. In the GPRD database, $9 \%$ of cases and $7 \%$ of controls had a history of diagnosed migraine $(\mathrm{OR}=1.3,95 \% \mathrm{CI}$ : $1.3-$ 1.4). The differential prevalences between the databases likely reflect between-country differences in diagnostics, awareness and treatment of migraine.

The current medication data was only reported for the patients with epilepsy to determine the most commonly used medications among patients with epilepsy. The most common AEDs varied between the US and UK databases. This is likely due to different treatment patterns and guidelines in the two countries. It has previously been shown that there is an association between the first AED prescribed after diagnosis of and history of psychiatric disorders, including depression and bipolar disorder, and the differential prescribing varied between the US and UK (Ishihara et al., 2010). One potential use of these data is to characterise polypharmacy patterns to inform potential drug interaction studies. Since the majority of patients are prescribed AEDs, the list identifies other medication types that are commonly used among the epilepsy patients.

The study should be interpreted in the context of its strengths and potential limitations. The strengths of the study include the large sizes of the databases, and the availability of detailed diagnosis and medication records. The GPRD is representative of the general population of the UK, and the IHCIS is representative of the US insured population. The IHCIS database is a large sample of people in the US covered by private insurance plans. Therefore results of this study may not be representative of US patients who receive healthcare through government organizations (e.g., Medicare, Medicaid) or who lack health insurance; for instance, these patients may be more likely to receive generic or older AEDs. In both databases, only diagnosed diseases are recorded, and therefore patients had to 
present to healthcare to be diagnosed with conditions or prescribed medications. There is a possibility of confounding by consultation frequency because patients with epilepsy or other chronic medical conditions will be more likely to be seen by healthcare providers compared to the general population.

The results of this study can be used to generate hypotheses about which medical conditions may be associated with epilepsy (either as risk factors for epilepsy, or resulting from epilepsy). Understanding the comorbidities and medication patterns in epilepsy also informs the effects of eligibility criteria on clinical trial recruitment, and facilitates assessments of potential safety signals that may arise in clinical trials (e.g. if a conditions is more common within the disease population at baseline, then this should be taken into account in evaluating whether exposure to a medication is associated with that condition).

\section{Conclusion}

In conclusion, psychiatric, neurological and other comorbidities are more common in patients with epilepsy, compared to age-, sex-matched controls. The results of this study provide hypotheses about conditions which may be associated with epilepsy, and these may be the subject for further investigation.

Among patients with epilepsy, the most commonly prescribed medications varied between the UK and US. Two of the older AEDs, valproate and carbamazepine, were the most commonly prescribed AEDs in 2009 amongst epilepsy patients in GPRD. Lamotrigine and phenytoin were the most commonly prescribed AEDs to patients with epilepsy in 2009 within IHCIS.

The most commonly prescribed medications among the epilepsy patient population, aside from AEDs, included analgesics, non-steroidal anti-inflammatory drugs, extended spectrum macroli (anti-biotics), selective serotonin reuptake inhibitors, and statins. The list of commonly prescribed medications in the epilepsy patient population can give an indication as to which drugs to consider for possible drug-drug interactions.

\section{References}

Baker, G.A., Jacoby, A., Chadwick, D.W. (1996). The associations of psychopathology in epilepsy: a community study. Epilepsy Res. Vol. 25, No. 1, pp. 29-39

Banerjee PN, Filippi D, Hauser WA. The descriptive epidemiology of epilepsy - a review. Epilepsy Res. 2009; 85: 31-45.

Beghi, E., Spagnoli, P., Airoldi, L., Fiordelli, E., Appollonio, I., Bogliun, G., et al. (2002). Emotional and affective disturbances in patients with epilepsy. Epilepsy Behav. Vol. 3, No. 3, pp. 255-261

Boylan, L.S., Flint, L.A., Labovitz, D.L., Jackson, S.C., Starner, K., Devinsky, O. (2004). Depression but not seizure frequency predicts quality of life in treatment-resistant epilepsy. Neurology. Vol. 62, No. 2, pp.258-261

Bredkjaer, S.R., Mortensen, P.B., Parnas, J. (1998). Epilepsy and non-organic non-affective psychosis. National epidemiologic study. Br J Psychiatry. Vol. 172, No. 3, pp. 235238 
Cramer, J.A., Blum, D., Fanning, K., Reed, M. (2004) The impact of comorbid depression on health resource utilization in a community sample of people with epilepsy. Epilepsy Behav. Vol. 5, No. 3, pp. 337-342

de Boer, H.M., Mula, M., \& Sander, J.W. (2008). The global burden and stigma of epilepsy. Epilepsy Behav. Vol. 12, No.4, pp.540-546

Edeh, J., Toone, B. (1987). Relationship between interictal psychopathology and the type of epilepsy. Results of a survey in general practice. Br J Psychiatry. Vol. 151, No.1, pp. 95-101.

Forsgren, L., Nystrom, L. (1990). An incident case-referent study of epileptic seizures in adults. Epilepsy Res. Vol. 6, No. 1, pp. 66-81.

Gaitatzis, A., Carroll, K., Majeed, A., Sander, J.W. (2004)(2). The epidemiology of the comorbidity of epilepsy in the general population. Epilepsia. Vol. 45, No. 12, pp. 1613-1622

Gaitatzis, A., Trimble, M.R., Sander, J.W. (2004). The psychiatric comorbidity of epilepsy. Acta Neurol Scand. Vol. 110, No. 4, pp. 207-220.

Glosser, G., Zwil, A.S., Glosser, D.S., O'Connor, M.J., Sperling, M.R. (2000). Psychiatric aspects of temporal lobe epilepsy before and after anterior temporal lobectomy. $J$ Neurol Neurosurg Psychiatry. Vol. 68, No. 1, pp. 53-58.

Gudmundsson, G. (1966). Epilepsy in Iceland. A clinical and epidemiological investigation. Acta Neurol Scand Vol. 43, Supp. L, p. 124.

Healthcare Cost and Utilization Project (HCUP, 2011). HCUP Clinical Classifications Software (CCS). Agency for Healthcare Research and Quality, Rockville, MD. Internet Citation: www.hcup-us.ahrq.gov/toolssoftware/ccs/ccs.jsp

Hermann, B.P., Seidenberg, M., Haltiner, A., Wyler, A.R. (1991). Mood state in unilateral temporal lobe epilepsy. Biol Psychiatry. Vol. 30, No. 12, pp. 1205 - 1218.

Hesdorffer, D.C., Hauser, W.A., Annegers, J.F., Cascino, G. (2000). Major depression is a risk factor for seizures in older adults. Ann Neurol. Vol. 47, No. 2, pp. 246 - 249.

Hesdorffer, D.C., Hauser, W.A., Olafsson, E., Ludvigsson, P., \& Kjartansson, O. (2006). Depression and suicide attempt as risk factors for incident unprovoked seizures. Ann Neurol. Vol. 59, No. 1, pp. 35-41

Ishihara L, Webb DJ, Irizarry M, Weil J. (2010) Exploring differential prescribing between anti-epileptic drugs in epilepsy patients with a history of mood disorders. Pharmacoepidemiol Drug Saf Vol. 19, No. 2, pp.289-295.

Jacoby, A., Baker, G.A., Steen, N., Potts, P., Chadwick, D.W. (1996). The clinical course of epilepsy and its psychosocial correlates: findings from a U.K. Community study. Epilepsia Vol. 37, No. 2, pp.148-161.

Jick, S.S., Kaye, J.A., Vasilakis-Scaramozza, C, Garcia Rodriguez, L.A., Ruigomez, A., Meier, C.R., Schlienger, R.G., Jick, J.(2003). Validity of the general practice research database. Pharmacotherapy; Vol. 23, No. , pp. 686-689.

Kanner, A.M. (2009). Suicidality and epilepsy: a complex relationship that remains misunderstood and underestimated. Epilepsy Curr. Vol. 9, No. 3, pp. 63 - 66.

Karaali-Savrun, F., Goksan, B., Yeni, S.N., Ertan, S., Uzun, N. (2002). Seizure-related headache in patients with epilepsy. Seizure. Vol. 11, No. 1, pp. 67-69. 
Kobau, R., Gilliam, F., Thurman, D.J. (2006). Prevalence of self-reported epilepsy or seizure disorder and its associations with self-reported depression and anxiety: results from the 2004 HealthStyles Survey. Epilepsia. Vol. 47, No. 11, pp. 1915-1921.

Lipton, R.B., Bigal, M.E., Diamond, M., Freitag, F., Reed, M.L., Stewart, W.F. (2007). Migraine prevalence, disease burden, and the need for preventive therapy. Neurology. Vol. 68, No. 5, pp. 343-349.

Lipton, R.B., Ottman, R., Ehrenberg, B.L., Hauser, W.A. (1994). Comorbidity of migraine: the connection between migraine and epilepsy. Neurology. Vol. 44, No. 10 Suppl 7, pp. S28-S32.

Marks, D.A., Ehrenberg, B.L. (1993). Migraine-related seizures in adults with epilepsy, with EEG correlation. Neurology. Vol. 43, No. 12, pp. 2476-2483.

Mendez, M.F., Cummings, J.L., Benson, D.F. (1986). Depression in epilepsy. Significance and phenomenology. Arch Neurol. Vol. 43, No. 8, pp. 766-770.

Nilsson, F.M., Kessing, L.V., Bolwig, T.G. (2003). On the increased risk of developing lateonset epilepsy for patients with major affective disorder. J Affect Disord. Vol. 76, No. 1-3, pp. 39-48.

Nuyen, J., Schellevis, F.G., Satariano, W.A., Spreeuwenberg, P.M., Birkner, M.D., van den Bos, G.A., et al. (2006) Comorbidity was associated with neurologic and psychiatric diseases: a general practice-based controlled study. J Clin Epidemiol. Vol. 59, No. 12, pp. 1274-1284.

O'Donoghue, M.F., Goodridge, D.M., Redhead, K., Sander, J.W., Duncan, J.S. (1999) Assessing the psychosocial consequences of epilepsy: a community-based study. Br J Gen Pract. Vol. 49, No. 440, pp. 211-214.

Ottman, R., Lipton, R.B. (1994). Comorbidity of migraine and epilepsy. Neurology. Vol. 44, No. 11, pp. 2105-2110

Piccinelli, P., Borgatti, R., Nicoli, F., Calcagno, P., Bassi, M.T., Quadrelli, M., et al. (2006). Relationship between migraine and epilepsy in pediatric age. Headache. Vol. 46, No. 3, pp. 413-421.

Shukla, G.D., Srivastava, O.N., Katiyar, B.C., Joshi, V., Mohan, P.K. (1979). Psychiatric manifestations in temporal lobe epilepsy: a controlled study. Br J Psychiatry. Vol. 135, No. 5, pp. 411-417.

Strine, T.W., Kobau, R., Chapman, D.P., Thurman, D.J., Price, P., Balluz, L.S. (2005). Psychological distress, comorbidities, and health behaviors among U.S. adults with seizures: results from the 2002 National Health Interview Survey. Epilepsia. Vol. 46, No. 7, pp. 1133-1139.

Tellez-Zenteno, J.F., Patten, S.B., Jette, N., Williams, J., Wiebe, S. (2007). Psychiatric comorbidity in epilepsy: a population-based analysis. Epilepsia. Vol. 48, No. 12, pp. 2336-2344.

Tomson, T., Beghi, E., Sundqvist, A., Johannessen, S.I. (2004). Medical risks in epilepsy: a review with focus on physical injuries, mortality, traffic accidents and their prevention. Epilepsy Res; Vol. 60 No. 1; pp. 1-16.

Toone, B. (2000). The psychoses of epilepsy. J Neurol Neurosurg. Vol. 69, No. 1, pp. 1-4

Torta, R., Keller, R. (1990). Behavioral, psychotic, and anxiety disorders in epilepsy: etiology, clinical features, and therapeutic implications. Epilepsia Vol. 40, Suppl. 10, pp. S2-20. 


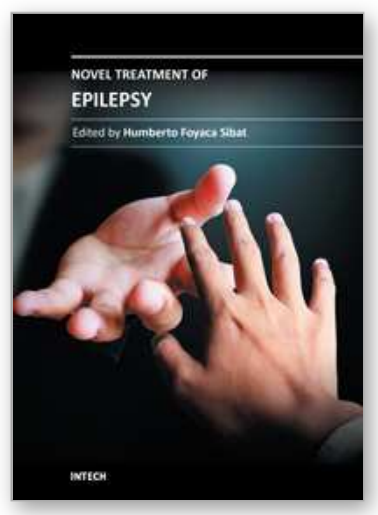

\author{
Novel Treatment of Epilepsy \\ Edited by Prof. Humberto Foyaca-Sibat
}

ISBN 978-953-307-667-6

Hard cover, 326 pages

Publisher InTech

Published online 22, September, 2011

Published in print edition September, 2011

Epilepsy continues to be a major health problem throughout the planet, affecting millions of people, mainly in developing countries where parasitic zoonoses are more common and cysticercosis, as a leading cause, is endemic. There is epidemiological evidence for an increasing prevalence of epilepsy throughout the world, and evidence of increasing morbidity and mortality in many countries as a consequence of higher incidence of infectious diseases, head injury and stroke. We decided to edit this book because we identified another way to approach this problem, covering aspects of the treatment of epilepsy based on the most recent technological results â€œin vitroâ€ from developed countries, and the basic treatment of epilepsy at the primary care level in rural areas of South Africa. Therefore, apart from the classic issues that cannot be missing in any book about epilepsy, we introduced novel aspects related with epilepsy and neurocysticercosis, as a leading cause of epilepsy in developing countries. Many experts from the field of epilepsy worked hard on this publication to provide valuable updated information about the treatment of epilepsy and other related problems.

\title{
How to reference
}

In order to correctly reference this scholarly work, feel free to copy and paste the following:

Lianna Ishihara and Michael Irizarry (2011). Co-Morbidity and Medication Profiles of Patients with Epilepsy and Matched Controls in US and UK Electronic Health Records Systems, Novel Treatment of Epilepsy, Prof. Humberto Foyaca-Sibat (Ed.), ISBN: 978-953-307-667-6, InTech, Available from: http://www.intechopen.com/books/novel-treatment-of-epilepsy/co-morbidity-and-medication-profiles-ofpatients-with-epilepsy-and-matched-controls-in-us-and-uk-ele

\section{INTECH}

open science | open minds

\section{InTech Europe}

University Campus STeP Ri

Slavka Krautzeka 83/A

51000 Rijeka, Croatia

Phone: +385 (51) 770447

Fax: +385 (51) 686166

www.intechopen.com

\section{InTech China}

Unit 405, Office Block, Hotel Equatorial Shanghai

No.65, Yan An Road (West), Shanghai, 200040, China 中国上海市延安西路65号上海国际贵都大饭店办公楼 405 单元

Phone: +86-21-62489820

Fax: $+86-21-62489821$ 
(C) 2011 The Author(s). Licensee IntechOpen. This chapter is distributed under the terms of the Creative Commons Attribution-NonCommercialShareAlike-3.0 License, which permits use, distribution and reproduction for non-commercial purposes, provided the original is properly cited and derivative works building on this content are distributed under the same license. 